\title{
Influence of PEGylation and RGD loading on the targeting properties of radiolabeled liposomal nanoparticles
}

This article was published in the following Dove Press journal:

International Journal of Nanomedicine

26 November 2012

Number of times this article has been viewed

\author{
Christine Rangger ${ }^{1}$ \\ Anna Helbok' \\ Elisabeth von Guggenberg' \\ Jane Sosabowski \\ Thorsten Radolf ${ }^{3}$ \\ Ruth Prassl ${ }^{4}$ \\ Fritz Andreae 3 \\ Gudrun C Thurner ${ }^{5}$ \\ Roland Haubner \\ Clemens Decristoforo' \\ 'Department of Nuclear Medicine, \\ Innsbruck Medical University, \\ Innsbruck, Austria; ${ }^{2}$ Center for \\ Molecular Oncology, Barts Cancer \\ Institute, Queen Mary University \\ of London, London, UK; ${ }^{3}$ piCHEM \\ Research and Development, \\ Graz, ${ }^{4}$ Institute of Biophysics and \\ Nanosystems Research, Austrian \\ Academy of Sciences, Graz, \\ ${ }^{5}$ Department of Radiology, Innsbruck \\ Medical University, Innsbruck, Austria
}

Correspondence: Clemens Decristoforo Universitaetsklinik fur Nuklearmedizin, Medizinische Universitaet Innsbruck, Anichstrasse 35, 6020 Innsbruck, Austria Tel +435125048095 I

Fax +435I2504678095I

Email clemens.decristoforo@uki.at
Purpose: Liposomes have been proposed to be a means of selectively targeting cancer sites for diagnostic and therapeutic applications. The focus of this work was the evaluation of radiolabeled PEGylated liposomes derivatized with varying amounts of a cyclic arginyl-glycyl-aspartic acid (RGD) peptide. RGD peptides are known to bind to $\alpha_{\mathrm{v}} \beta_{3}$ integrin receptors overexpressed during tumor-induced angiogenesis.

Methods: Several liposomal nanoparticles carrying the RGD peptide targeting sequence (RLPs) were synthesized using a combination of 1-palmitoyl-2-oleoyl-sn-glycero-3-phosphocholine, cholesterol, diethylenetriaminepentaacetic acid-derivatized lipids for radiolabeling, a polyethylene glycol (PEG) building block, and a lipid-based RGD building block. Relative amounts of RGD and PEG building blocks were varied. In vitro binding affinities were determined using isolated $\alpha_{\mathrm{v}} \beta_{3}$ integrin receptors incubated with different concentrations of RLPs in competition with iodine-125-labeled cyclo-(-RGDyV-). Binding of the indium-111labeled RLPs was also evaluated. Biodistribution and micro single photon emission computed tomography/computed tomography imaging studies were performed in nude mice using different tumor xenograft models.

Results: RLPs were labeled with indium-111 with high radiochemical yields. In vitro binding studies of RLPs with different RGD/PEG loading revealed good binding to isolated receptors, which was dependent on the extent of RGD and PEG loading. Binding increased with higher RGD loading, whereas reduced binding was found with higher PEG loading. Biodistribution showed increased circulating time for PEGylated RLPs, but no dependence on RGD loading. Both biodistribution and micro single photon emission computed tomography/computed tomography imaging studies revealed low, nonspecific tumor uptake values.

Conclusion: In this study, RLPs for targeting angiogenesis were described. Even though good binding to $\alpha_{\mathrm{v}} \beta_{3}$ integrin receptors was found in vitro, the balance between PEGylation and RGD loading clearly requires optimization to achieve targeting in vivo. These data form the basis for future development and provide a platform for the investigation of multimodal approaches.

Keywords: liposomes, RGD peptides, $\alpha_{\mathrm{v}} \beta_{3}$ integrin receptors, angiogenesis, tumor targeting

\section{Introduction}

Since their first characterization in 1965 by Bangham et al, ${ }^{1}$ liposomes have been under extensive investigation. They consist of one or more concentric lipid bilayers with varying size and are unique in their ability to encapsulate and store drugs that differ widely in terms of their physicochemical properties, eg, polarity, charge, and size. ${ }^{2}$ These drugs include chemotherapeutic agents, antibiotics, antigens, fungicides, contrast agents, chelating compounds, and radionuclides, which are incorporated into the liposome's aqueous interior or bound within or onto the surface of the lipid membrane. ${ }^{3}$ A number of 
anticancer liposomal drug delivery systems have already been approved for clinical use., ${ }^{4,5}$

Notwithstanding their usefulness as carriers for various drugs, a major disadvantage of the in vivo use of liposomal nanoparticles (LNPs) is their recognition by phagocytic cells of the mononuclear phagocyte system. This leads to their rapid removal from the blood circulation into mainly the liver and spleen. Workers in the field have conducted extensive investigations into the effects of particle size, lipid composition, and surface charge of the liposome in order to find a solution to this problem. ${ }^{6}$ One observation of the many experiments conducted was that polyethylene glycol (PEG), a synthetic hydrophilic polymer introduced in the 1970s, improves the pharmacokinetics of liposomes by imparting a steric barrier to the surface of the liposome, thus minimizing opsonization (a process whereby the liposome is marked for destruction via phagocytosis). This reduces recognition by the Kupffer cells of the liver via the scavenger receptor. ${ }^{7}$ PEGylation has also been found to increase biological stability, to reduce leakage of the encapsulated drug, ${ }^{8}$ and to prevent aggregation, thus aiding the formation of small, monodispersed particles. ${ }^{9}$ Much research in this field has been carried out to evaluate, for example, the influence of PEG loading or PEG chain length, 7,10 and a number of PEGylated liposomes have obtained approval for clinical use. ${ }^{11}$

In contrast to the above-mentioned limitations, LNPs benefit from the enhanced permeability and retention effect where the long circulation time of nanoparticles allows accumulation in tissues with compromised vasculature, eg, areas of inflammation or tumors. ${ }^{12}$ The increased microvascular permeability of a tumor, characterized by the absence of a basal membrane and the presence of gaps between the endothelial cells, allows nanoparticles to extravasate from the general circulation. Moreover, the absence of lymphatic vessels promotes accumulation within the tumor environment, causing so-called "passive targeting." "13 The drawback with this route of accumulation is the lack of cell-specific interaction, which reduces the internalization of the LNP and its encapsulated drugs. The therapeutic efficacy of the liposomal carrier is therefore minimized. ${ }^{14}$ As a consequence, the main challenge in this field is to combine passive with active targeting using targetspecific sequences interacting with receptors on the cell surface. This will result in a higher concentration of drug at the tumor site, which will increase the potency of treatments and reduce possible side effects.

In the current study, a cyclic arginyl-glycyl-aspartic acid (RGD; cyclo-[-Arg-Gly-Asp-DTyr-Val-]) peptide was used as a target-specific sequence. RGD peptides are active modulators of cell adhesion and can specifically bind to the well-studied $\alpha_{v} \beta_{3}$ integrin receptors. These receptors are expressed during tumor-induced angiogenesis of osteoclasts and are found in invasive tumors such as latestage glioblastomas, breast and prostate cancer, malignant melanomas, and ovarian carcinomas. ${ }^{15}$ The formation of new blood vessels is essential for these tumors to supply them with sufficient oxygen and nutrients. The activated endothelium of such angiogenic blood vessels strongly expresses $\alpha_{\mathrm{v}} \beta_{3}$ integrin receptors. Most importantly, these $\alpha_{\mathrm{v}} \beta_{3}$ integrin receptors are only weakly expressed on endothelial cells in nondiseased tissue, making it a specific target for antiangiogenic therapy monitoring, ${ }^{16,17}$ improved prognostic evaluation of cancer aggressiveness, ${ }^{18}$ and tumor angiogenesis imaging. ${ }^{19,20}$ Therefore, using RGD peptides as integrin antagonists targeting the tumor microenvironment might curtail tumor progression, ${ }^{21}$ and could also be applied as a noninvasive tool to visualize angiogenesis in growing tumors.

In order to perform noninvasive imaging, LNPs can be labeled with metallic radionuclides to follow their in vivo fate using nuclear medicine imaging modalities such as single photon emission computed tomography (SPECT) or positron emission tomography. For this purpose, a variety of radionuclides are used, eg, indium-111 ( $\left.{ }^{111} \mathrm{In}\right)$, gallium-68, technetium-99m, fluorine-18, rhenium-188, or copper-64. ${ }^{22-25}$ In addition to using LNPs for diagnostic imaging, they have also been labeled with radium-223, actinium-225, lutetium-177, or yttrium-90 to investigate their potential for use in radionuclide therapy in cancer patients. $^{22,26-30}$ The advantage in using radioisotope imaging of LNPs is that these techniques are extremely sensitive and an absolute quantification of the compound is possible. ${ }^{31}$ The radiolabeling of LNPs using a diethylenetriaminepentaacetic acid (DTPA)-derivatized lipid building block - which allows radiolabeling of liposomes with high specific activity and high stability in vitro and in vivo, respectively - has recently been investigated. ${ }^{22}$

Here, the different ${ }^{111}$ In-labeled LNPs, which differ in their RGD and PEG loading, were evaluated and the influence of these surface modifications on the pharmacokinetics of the liposome was investigated. Additionally, the radiolabeling properties and the in vitro and in vivo fate of different liposomes derivatized with an RGD peptide (RLPs) for targeting and noninvasive imaging of angiogenesis in two tumor xenograft models in nude mice were investigated. Furthermore, these RLPs were used for micro-SPECT/CT imaging studies to 
explore their future usage in cancer diagnosis and therapy as well as for multimodal imaging.

\section{Material and methods}

Unless stated otherwise, all reagents were of analytical grade obtained from VWR International (Radnor, PA) or SigmaAldrich (St Louis, MO) and used as supplied with no further purifications.

1-palmitoyl-2-oleoyl-sn-glycero-3-phosphocholine and 1,2-dimyristoyl-sn-glycero-3-phosphoethanolamineN-DPTA were obtained from Avanti Polar Lipids, Inc (Alabaster, AL), together with the Avanti ${ }^{\circledR}$ Mini-Extruder for liposome preparation. Cholesterol was purchased from Sigma-Aldrich. ${ }^{111}$ In chloride was obtained either from PerkinElmer (Waltham, MA) or Mallinckrodt Medical BV (Petten, The Netherlands) and iodine-125 ( $\left.{ }^{125} \mathrm{I}\right)$ from GE Healthcare (Little Chalfont, UK). The human $\alpha_{\mathrm{v}} \beta_{3}$ integrin receptor was purchased from EMD Millipore (Billerica, MA).

Cell culture media (Gibco ${ }^{\circledR}$ Dulbecco's modified Eagle's medium [DMEM], Gibco Roswell Park Memorial Institute 1640 [RPMI 1640]; Life Technologies, Carlsbad, CA), Gibco fetal bovine serum (Life Technologies), penicillin/ streptomycin/glutamine solution (100X, liquid), sodium pyruvate solution (100 mM, liquid), minimum essential medium nonessential amino acid solution (100X, liquid), and trypsin solution $(2.5 \%, 10 \mathrm{X}$, liquid) were purchased from Thermo Fisher Scientific (Waltham, MA).

Human melanoma cells (M21 and M21-L) were a kind gift from Dr David A Cheresh and the Scripps Research Institute (La Jolla, CA). Human glioblastoma astrocytoma cells (U-87 MG) were purchased from the European Collection of Cell Cultures (Salisbury, UK).

Radioactivity of the samples was measured using a 2480 Wizard $^{2}$ Automatic Gamma Counter (PerkinElmer).

\section{Preparation of the RGD building block Synthesis of S-acetyl-3-mercaptopropionic acid} (SAMA)-RGD

Synthesis of SAMA-RGD was performed using the solid phase peptide synthesis technique with a commercial batch synthesizer using a preloaded 9-fluorenylmethyloxycarbonyl-Gly-2-Cl-trityl resin and hydroxybenzotriazole/ diisopropylcarbodiimide in situ activation. The linear side chain protected peptide was cleaved from the resin using a mixture of $20 \%$ 1,1,1,3,3,3-Hexafluoro-2-propanol in dichloromethane. The crude product $(\mathrm{H}-\mathrm{Asp}[\mathrm{OtBu}]$ DTyr[tBu]-Lys[1-[4,4-dimethyl-2,6-dioxocyclohexylidene]
ethyl]-Arg[Pbf]-Gly-OH) was then purified by high-performance liquid chromatography (HPLC) and freeze dried, followed by carbon-nitrogen cyclization of the linear protected peptide in acetonitrile by use of benzotriazol-1-yl-oxytris-pyrrolidino-phosphonium hexafluorophosphate. After removal of the 1-(4,4-dimethyl-2,6-dioxocyclohexylidene) ethyl protection group of lysine using $2 \%$ hydrazine hydrate in 1-methyl-2-pyrrolidinone, the crosslinker SAMA was attached onto the side chain amino group of lysine using preactivated SAMA pentafluorophenyl ester. Each cleavage, cyclization, or modification step was finalized by reversedphase HPLC purification and lyophilization.

Crosslinking of I,2-distearoyl-sn-glycero-3phosphoethanolamine-N-(maleimide[PEG]-2000) (DSPE-PEG[2000]-Mal) and SAMA-RGD

The crosslinking of SAMA-RGD with DSPE-PEG(2000)Mal was performed by in situ deprotection of the S-acetyl group with hydroxylamine, releasing a free thiol moiety which then reacts with the maleimide group of DSPE-PEG(2000)Mal. Briefly, SAMA-RGD and DSPE-PEG(2000)-Mal were dissolved in methanol at a molar peptide-to-lipid ratio of 1.5:1 $\left(5.4 \times 10^{-6} \mathrm{~mol}\right.$ SAMA-RGD:3.6 $\times 10^{-6} \mathrm{~mol}$ DSPEPEG[2000]-Mal). After the addition of a 200 -fold molar excess of hydroxylamine over SAMA-RGD dissolved in $0.1 \mathrm{M}$ phosphate-buffered saline ( $\mathrm{pH}$ 6.0), the mixture was left to react for 4 hours at room temperature and was finally freeze dried. Completeness of product formation was monitored by reversed-phase HPLC and mass spectrometry. Residual amounts of unreacted SAMA-RGD were removed by dissolving the RGD building block in chloroform and the product was again monitored by reversed-phase HPLC, confirming a purity of more than $95 \%$.

\section{Preparation of RLPs}

RLPs were prepared by a thin layer rehydration technique using a ternary lipid mixture, which self-assembles to form sterically stabilized liposomes. Therefore, stock solutions of 1-palmitoyl-2-oleoyl-sn-glycero-3-phosphocholine, cholesterol, DSPE-PEG(2000), 1,2-dimyristoyl-sn-glycero-3phosphoethanolamine-N-DTPA, and the RGD building block were prepared and dissolved in pure chloroform in a round bottom glass vial. For the different liposome types, aliquots of the above-mentioned components (1-palmitoyl-2-oleoyl-snglycero-3-phosphocholine/cholesterol/DSPE-PEG(2000)/1,2dimyristoyl-sn-glycero-3-phosphoethanolamine-N-DTPA/ RGD) were mixed in a molar ratio of $3 / 2 / 0.3 / 0.3 / 0.003$ for low RGD loading (lowRLP; 0.06 mol\% RGD content), 
3/2/0.3/0.3/0.03 for medium RGD loading (medRLP; $0.6 \mathrm{~mol} \%$ RGD content), and 3/2/0.3/0.3/0.3 for high RGD loading (highRLP; 6 mol\% RGD), obtaining a total lipid content of $30 \mathrm{mg} / \mathrm{mL}$. In additional experiments, the relative amount of DSPE-PEG(2000) was varied in medRLP as follows: 0, 0.6, 3, and $6 \mathrm{~mol} \%$ PEG lipid. A standard liposome carrying no RGD building block (standardLP) was also prepared. The organic solvent (chloroform) was then evaporated under a stream of nitrogen yielding a thin lipid film on the side of the glass vial, which was allowed to dry under vacuum overnight. Hydration of the lipid film was achieved by adding $1 \mathrm{~mL}$ of $6.6 \mathrm{mM}$ disodium phosphate dihydrate/1.4 mM monopotassium phosphate/136.9 mM sodium chloride phosphate-buffered saline (pH 7.4) and repeatedly vortexed for 1 hour at $40^{\circ} \mathrm{C}$. The lipid suspensions were then extruded 21 times through a polycarbonate filter (EMD Millipore) with a pore size of $100 \mathrm{~nm}$ using the Avanti Mini-Extruder. The resulting liposomes were stored at $4^{\circ} \mathrm{C}$. The maximum storage time for liposomal suspensions was 1 month based on stability studies performed by the authors' group. ${ }^{22}$

\section{Characterization of RLPs}

The particle size distribution of RLPs was identified using photon correlation spectroscopy. The procedure was carried out with a Submicron Particle Sizer Nicomp ${ }^{\text {TM }} 380$ (Particle Sizing Systems, Port Richey, FL). This dynamic light scattering instrument is equipped with a $15 \mathrm{~mW}$ laser diode and a photomultiplier tube detector with an optical fiber set at 90 degrees to detect the scattered light from the probe. Particle size is derived by an autocorrelation function. Results were expressed as the Z-average, which is the harmonic intensity averaged particle diameter. The width of the size distribution is given by the polydispersity index. All measurements were performed at room temperature. Zeta potential measurements of the various liposomes were performed using a Nicomp 380 ZLS Particle Sizer (Particle Sizing Systems), at $23^{\circ} \mathrm{C}$ and an E-field strength of $5 \mathrm{mV}$. For the data acquisition, PSS ZPW388 version 1.65 software (Particle Sizing Systems) was used.

\section{Radiolabeling}

Based on the results of Helbok et al, ${ }^{22}$ the optimized standard labeling protocol was as follows: ${ }^{111}$ In chloride (5-100 MBq/mL) diluted in $0.05 \mathrm{M}$ hydrogen chloride to a volume of $50 \mu \mathrm{L}$ and $50 \mu \mathrm{L}$ of sodium acetate $(0.4 \mathrm{M}) /$ gentisic acid $(0.24 \mathrm{M})$ buffer $(\mathrm{pH} 4.5)$ were added to $100 \mu \mathrm{L}$ liposomal suspension, resulting in a total reaction volume of $200 \mu \mathrm{L}$. The labeling solution was allowed to react at room temperature for 30 minutes.
For the determination of radiolabeling efficiency (radiochemical yield), instant thin layer chromatography on silica gel strips (Agilent Technologies, Santa Clara, CA) was used. Samples were developed with two different mobile phases: (1) $1 \mathrm{M}$ ammonium acetate/0.05 M ethylenediaminetetraacetic acid, in which system labeled RLP and radiocolloid remain at the origin (retention factor radiolabeled RLP and radiocolloid $=0.0-0.3$ ) and "free" radionuclide migrates with the solvent front (retention factor "free" radionuclide $=0.8-1.0$ ); and (2) acetic acid/ pyridine/water (5/3/1.5), in which system labeled RLP and "free" radionuclide migrate with the solvent front (retention factor radiolabeled RLP and "free" radionuclide $=0.8-1.0$ ) and radiocolloids remain at the origin (retention factor radiocolloid $=0.0-0.2$ ). The distribution of radioactivity was analyzed by electronic autoradiography using the Cyclone ${ }^{\circledR}$ Plus Phosphor Imager (PerkinElmer).

\section{Evaluation of RLPs in vitro}

\section{Receptor binding studies}

Isolated human $\alpha_{\mathrm{v}} \beta_{3}$ integrin receptors were used to determine the in vitro binding affinities of various RLPs. The original method of Nachman and Leung was followed with some modifications. ${ }^{32}$ For this purpose two different approaches were used: (1) competition assay and (2) binding assay.

\section{Competition assay}

Binding affinities of RLPs (lowRLP, medRLP, highRLP, standardLP) were determined using ${ }^{125} \mathrm{I}$-cyclo-(-RGDyV-) as the radioligand, radio iodinated using the iodogen method of Fraker and Speck. ${ }^{33}$ The day before the experiment, 96-well Nunc $^{\circledR}$ MaxiSorp ${ }^{\mathrm{TM}}$ plates (Thermo Fisher) were coated with the isolated $\alpha_{\mathrm{v}} \beta_{3}$ integrin receptors (10 ng receptor/ well) diluted in coating buffer (25 $\mathrm{mM}$ tris[hydroxymethyl] aminomethane, $150 \mathrm{mM}$ sodium chloride, $1 \mathrm{mM}$ calcium chloride, $0.5 \mathrm{mM}$ magnesium chloride, $10 \mu \mathrm{M}$ manganese[II] chloride tetrahydrate). The receptors were left to incubate for 16 hours at $4{ }^{\circ} \mathrm{C}$, followed by 2 hours of incubation with blocking buffer (coating buffer plus 1\% bovine serum albumin) to reduce nonspecific binding of RLP to the wells. Then to each well (in triplicate) $50 \mu \mathrm{L}$ of competitor (RLP) in increasing concentrations (0.01-10,000 nM RGD in binding buffer [coating buffer plus $0.1 \%$ bovine serum albumin]) and $50 \mu \mathrm{L}$ radioligand solution $\left(\sim 20,000 \mathrm{cpm}{ }^{125} \mathrm{I}\right.$-cyclo-(RGDyV-) in binding buffer; $<1 \mathrm{nM})$ was added. As a control assay, the same procedure was carried out using cyclo-(RGDyV-) as the competitor. The assay was stopped after 2 hours by removing the solutions. Each well was washed 
twice with $150 \mu \mathrm{L}$ ice-cold binding buffer. The remaining receptor bound activity was removed with $100 \mu \mathrm{L}$ hot $\left(\sim 60^{\circ} \mathrm{C}\right)$ $2 \mathrm{M}$ sodium hydroxide solution and transferred into plastic vials which were then measured in a gamma counter. The half-maximal inhibitory concentration values were calculated by fitting the data with nonlinear regression (Origin Software; OriginLab Corporation, Northampton, MA).

\section{Binding assay}

Similar to the above-mentioned assay 96-well Nunc MaxiSorp plates were coated with the isolated $\alpha_{\mathrm{v}} \beta_{3}$ integrin receptors (10 ng receptor/well) for 16 hours at $4^{\circ} \mathrm{C}$, followed by 2 hours of incubation with blocking buffer. RLPs (lowRLP, medRLP, highRLP, medRLP with $0,0.6,3$, and $6 \mathrm{~mol} \%$ PEG lipid) were labeled with ${ }^{111}$ In chloride and the labeled compounds were incubated with the receptors for 2 hours at room temperature. Additionally, each radiolabeled compound was incubated with $10 \mu \mathrm{M}$ cyclo-(-RGDyV-) to determine nonspecific binding to the wells. After 2 hours, unbound radioligand was removed, the plates were washed twice, and the receptor bound activity collected with hot $2 \mathrm{M}$ sodium hydroxide solution. Samples were then measured in a gamma counter.

\section{Evaluation of RLPs in vivo Cell culture}

Evaluation of RLPs in vivo was carried out using $\alpha_{\mathrm{v}} \beta_{3}$ integrin receptor expressing M21 cells, whereas M21-L cells, which do not express the receptor, acted as negative control. ${ }^{34}$ M21 cells were maintained in DMEM and M21-L cells in RPMI 1640, both cell culture media supplemented with $10 \%$ volume/volume $(\mathrm{v} / \mathrm{v})$ heat-inactivated fetal bovine serum and $1 \% \mathrm{v} / \mathrm{v}$ penicillin/streptomycin/glutamine solution. M21 cells were grown in tissue culture flasks (Cellstar ${ }^{\circledR}$; Greiner Bio-One, Kremsmuenster, Austria) and M21-L cells in germ count dishes (Greiner Bio-One). For imaging studies, U-87 MG cells - known to express $\alpha_{\mathrm{v}} \beta_{3}$ integrins - were used and cultured in DMEM supplemented with $10 \% \mathrm{v} / \mathrm{v}$ heat-inactivated fetal bovine serum, $1 \% \mathrm{v} / \mathrm{v}$ penicillin/streptomycin/glutamine solution, $1 \% \mathrm{v} / \mathrm{v}$ sodium pyruvate solution, and $1 \% \mathrm{v} / \mathrm{v}$ nonessential amino acid solution. All cell lines were grown to confluence at $37^{\circ} \mathrm{C}$ in a humidified atmosphere of $95 \%$ air $/ 5 \%$ carbon dioxide and split every 48 hours.

\section{Biodistribution studies}

All animal experiments were conducted in compliance with the Austrian animal protection laws and with the approval of the Austrian Ministry of Science (BMWF-66.011/0147-II/10b/2008).

Biodistribution studies were performed on 6-weekold female athymic BALB/c nude mice (Charles River Laboratories, Sulzfeld, Germany), which were maintained on a normal ad libitum diet under pathogen-free conditions. For the induction of tumor xenografts, M21 cells were injected subcutaneously at a concentration of $5 \times 10^{6}$ cells $/ 100 \mu \mathrm{L}$ into the right hind limb and as negative control $3.5 \times 10^{6}$ M21-L cells/100 $\mu \mathrm{L}$ into the left hind limb of the mouse. Tumor size and state of health of the animals was checked regularly. After about 3 weeks, tumor size was about $1 \mathrm{~cm}$ in diameter and mice were randomly selected and put into groups of three.

On the day of the experiment, mice were injected intravenously into a lateral tail vein with $0.3 \mathrm{MBq}(15 \mathrm{MBq} / \mathrm{kg}$ bodyweight ${ }^{111} \mathrm{In}$; injected volume: $150 \mu \mathrm{L}$ ) of different ${ }^{111}$ In-RLPs (lowRLP, medRLP, highRLP, medRLP with no PEG), corresponding to approximately $10 \mathrm{ng}$ RGD/mouse. During the incubation time, mice were kept warm to avoid hypothermia. At 1 hour or 4 hours postinjection, the animals were sacrificed by cervical dislocation without anesthesia by trained personnel using appropriate equipment. Organs (heart, lung, liver, spleen, pancreas, stomach, intestine, kidneys), tissue (blood, muscle), and tumors were removed and weighed. Subsequently, the content of the stomach and intestine were removed and the organs then weighed. The accumulated radioactivity in the different specimens was measured in a gamma counter and results were expressed as percentage of injected dose per gram of tissue $(\% \mathrm{ID} / \mathrm{g})$.

\section{Micro-SPECT/CT imaging studies}

Imaging studies were carried out at the Center for Molecular Oncology, Barts Cancer Institute, Queen Mary University of London (London, UK).

About 2 weeks before the experiment, female beige severe combined immunodeficiency mice were injected subcutaneously into the left flank with $2.5 \times 10^{6} \mathrm{U}-87 \mathrm{MG}$ cells and tumors were left to grow until they reached a size of about $1 \mathrm{~cm}$ in diameter. Subsequently, approximately $12 \mathrm{MBq}$ of ${ }^{111} \mathrm{In}$-lowRLP, ${ }^{111} \mathrm{In}$ medRLP, and ${ }^{111}$ In-highRLP (injected volume: $200 \mu \mathrm{L}$ ) was administered via a lateral tail vein of the mouse. After 4, 24, 48, and 96 hours, mice were anesthetized (4\% isoflurane and $0.5-1 \mathrm{~L} /$ minute oxygen) and images were obtained using a NanoSPECT/CT in vivo preclinical imager four-head camera (Bioscan, Inc, Washington, DC) fitted with $2 \mathrm{~mm}$ pinhole collimators in helical scanning mode ( 20 projections, 30 minutes). The corresponding CT images were acquired 
with a $45 \mathrm{kVp}$ X-ray source over 10 minutes. Images were then reconstructed in a $256 \times 256$ matrix using InVivoScope ${ }^{\mathrm{TM}}$ software (Bioscan) and fused using PMOD $^{\mathrm{TM}}$ (Mediso Medical Imaging Systems, Budapest, Hungary).

\section{Statistics}

All data were expressed as the mean \pm standard deviation. Data of the in vitro assays and the biodistribution studies in tumor-xenografted BALB/c nude mice were compared using an unpaired $t$-test with a significance level of $P<0.01$. All analyses were performed using Microsoft Office XP Professional Excel ${ }^{\circledR}$ version 10.0.6626.0 (Microsoft Corporation, Redmond, WA).

\section{Results}

\section{Characterization and radiolabeling of RLPs}

The particle size distribution was highly homogenous for all tested RLPs. Results were reproducible and the mean diameter of the liposomes was $92.1 \pm 3.1 \mathrm{~nm}$ for lowRLP $(\mathrm{n}=9), 99.2 \pm 2.4 \mathrm{~nm}$ for medRLP $(\mathrm{n}=7)$, and $110.4 \pm 4.3 \mathrm{~nm}$ for highRLP $(\mathrm{n}=5)$. The polydispersity index values of the different liposomal suspensions were $0.006-0.2$. The zeta potential measurements of the various liposome populations revealed values between $-1.8 \mathrm{mV}$ and $6.3 \mathrm{mV}$.

All different RLPs could be labeled with ${ }^{111}$ In chloride and high radiochemical yield values $(>95 \%)$ were reached even using small amounts of RLP. The following radiochemical yield values were obtained: $98.3 \% \pm 3.0 \%$ for lowRLP $(n=9), 99.4 \% \pm 0.7 \%$ for medRLP $(n=12)$, and $97.6 \% \pm 1.7 \%$ for highRLP $(n=6)$. Radiochemical yield values were not influenced by lipid composition and particle size, but deteriorated over time to values below $80 \%$ using liposome solutions older than 6 months. Therefore, freshly prepared liposomes ( $<1$ month storage) were used throughout the experiments.

\section{Evaluation of RLPs in vitro}

\section{Receptor binding studies}

\section{Competition assay}

The in vitro binding characteristics of RLPs (lowRLP, medRLP, highRLP, standardLP) as well as cyclo-(-RGDyV-) as a reference substance were tested in competition with ${ }^{125} \mathrm{I}$-cyclo-(-RGDyV-) on isolated $\alpha_{\mathrm{v}} \beta_{3}$ integrin receptors. The amount of cell-bound radioactivity (percentage of total activity) was plotted against the amount of lipid/mL of liposome solution (the corresponding RGD values were calculated in $\mu \mathrm{g}$ lipid/mL) as shown in Figure 1. The halfmaximal inhibitory concentration values found in this experiment were $609.4 \mu \mathrm{g}$ lipid/mL for lowRLP, $37.8 \mu \mathrm{g}$ lipid/mL for medRLP, and $6.0 \mu \mathrm{g}$ lipid/mL for highRLP compared to $2570.0 \mu \mathrm{g}$ lipid/mL for standardLP, showing a clear effect of RGD loading on the inhibition of $\alpha_{v} \beta_{3}$ integrin binding of the standard radioligand ${ }^{125} \mathrm{I}$-cyclo-(-RGDyV-).

\section{Binding assay}

The differences in the $\alpha_{\mathrm{v}} \beta_{3}$ integrin receptor binding of ${ }^{111}$ In-labeled RLPs (lowRLP, medRLP, highRLP, medRLP with $0,0.6,3$, and $6 \mathrm{~mol} \% \mathrm{PEG}$ ) were evaluated in a similar manner to the above-mentioned competition assay. These studies showed that with higher RGD loading, binding to the $\alpha_{v} \beta_{3}$ integrin receptors increased from $1.6 \%$ of total activity for ${ }^{111}$ In-lowRLP to $2.2 \%$ for ${ }^{111}$ In-medRLP and $3.8 \%$ for ${ }^{111}$ In-highRLP (Figure 2). In addition, a detrimental effect of PEGylation on binding was shown. ${ }^{111}$ In-medRLP without PEGylation displayed the highest binding of the tested RLPs with a value of $13.3 \%$, whereas the same ${ }^{111}$ In-medRLP with 6 mol\% PEG demonstrated almost four-fold lower binding (3.4\% of total activity) (Figure 3 ).

\section{Evaluation of RLPs in vivo}

\section{Biodistribution studies}

Ex vivo distribution properties and receptor specific uptake of different ${ }^{111}$ In-labeled RLPs (lowRLP, medRLP, highRLP, medRLP without PEGylation) were studied at 1 hour and

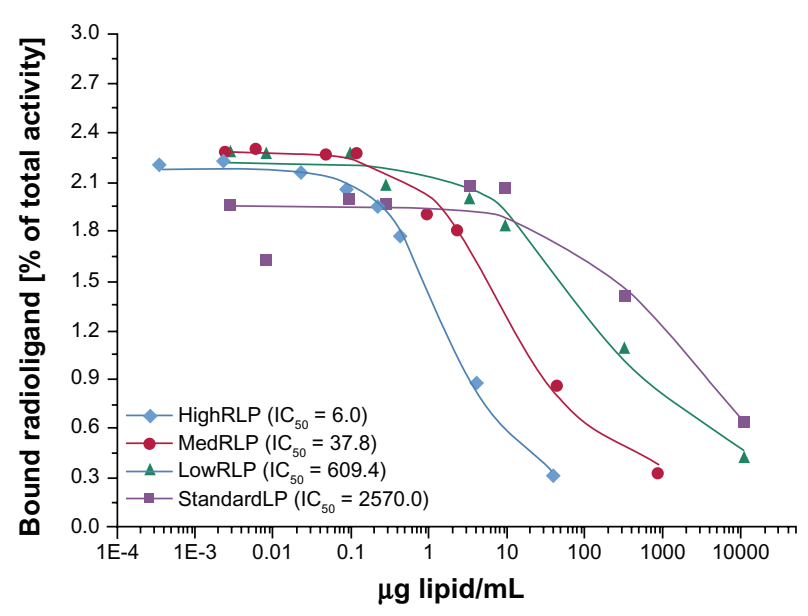

Figure I Binding affinities of lowRLP, medRLP, highRLP, and standardLP competing with iodine-125-labeled cyclo-(-RGDyV-) as the radioligand using isolated $\alpha_{v} \beta_{3}$ integrin receptors on 96 -well plates.

Note: Values are expressed as $\mathrm{IC}_{50}$.

Abbreviations: $I C_{50}$, half-maximal inhibitory concentration; lowRLP, RLP with low RGD loading; highRLP, RLP with high RGD loading; medRLP, RLP with medium RGD loading; RGD, arginyl-glycyl-aspartic acid; RLP, liposomal nanoparticles carrying an RGD building block; standardLP, standard liposome carrying no RGD building block. 


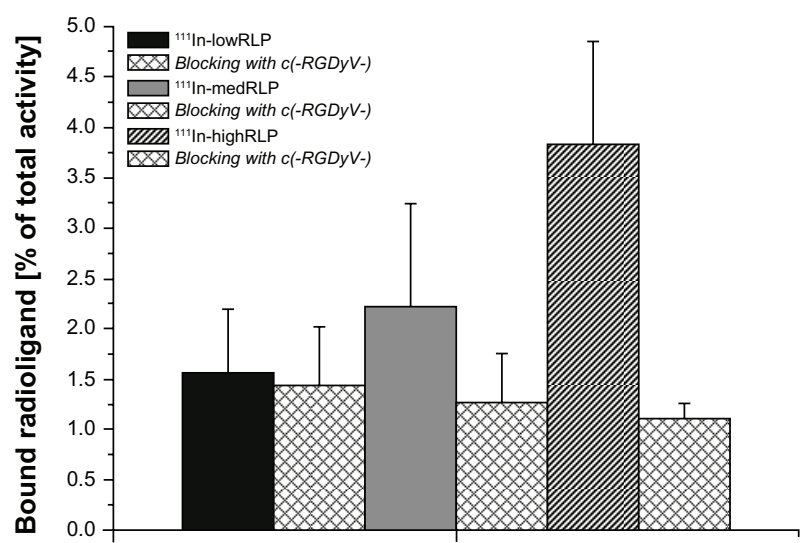

Figure 2 Comparison of in vitro binding characteristics of "'In-labeled lowRLP, medRLP, and highRLP on isolated $\alpha_{v} \beta_{3}$ integrin receptors using 96-well plates. Note: Values are expressed as mean \pm standard deviation.

Abbreviations: "'In, indium-I I ; lowRLP, RLP with low RGD loading; highRLP, RLP with high RGD loading; medRLP, RLP with medium RGD loading; RGD, arginyl-glycyl-aspartic acid; RLP, liposomal nanoparticles carrying an RGD building block.

4 hours postinjection in BALB/c nude mice bearing either the $\alpha_{\mathrm{v}} \beta_{3}$-positive M21 or the M21-L negative control tumor. The results are summarized in Table 1 .

For all RLPs, comparable results were found. The major uptake was in the spleen and liver, with the highest liver uptake values of $45.7 \% \pm 2.2 \% \mathrm{ID} / \mathrm{g}$ for ${ }^{111} \mathrm{In}$-medRLP and $32.9 \% \pm 5.7 \% \mathrm{ID} / \mathrm{g}$ for ${ }^{111} \mathrm{In}$-lowRLP, whereas for ${ }^{111} \mathrm{In}$-highRLP only $21.5 \% \pm 6.6 \% \mathrm{ID} / \mathrm{g}$ was seen at 1 hour postinjection. PEGylated RLPs displayed high blood levels ( ${ }^{111} \mathrm{In}-$ lowRLP: $18.1 \% \pm 2.9 \% \mathrm{ID} / \mathrm{g} ;{ }^{111} \mathrm{In}-\mathrm{medRLP}$ : $17.0 \% \pm 5.1 \% \mathrm{ID} / \mathrm{g} ;{ }^{111} \mathrm{In}$-highRLP: $10.0 \% \pm 4.7 \% \mathrm{ID} / \mathrm{g}$ ). Uptake values for the other tissues (heart, stomach, pancreas, intestine, kidneys) at 1 hour postinjection were rather low

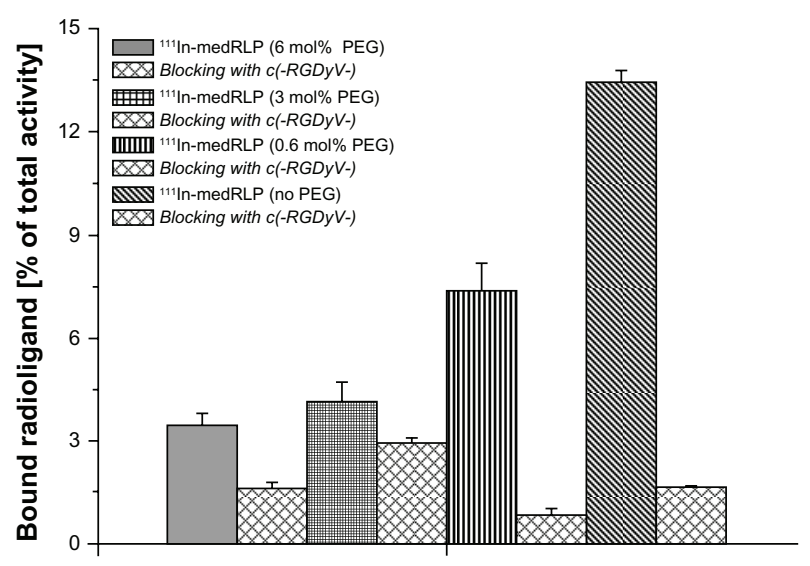

Figure 3 In vitro binding assay of "'In-labeled medRLP with varying PEG amounts using immobilized $\alpha_{v} \beta_{3}$ integrin receptors on 96-well plates.

Note: Values are expressed as mean \pm standard deviation.

Abbreviations: 'I'In, indium-III; PEG, polyethylene glycol; medRLP, RLP with medium RGD loading; RGD, arginyl-glycyl-aspartic acid; RLP, liposomal nanoparticles carrying an RGD building block.
$(<9.0 \% \mathrm{ID} / \mathrm{g})$, with the exception of lungs where the accumulation was $13.1 \% \pm 1.9 \% \mathrm{ID} / \mathrm{g}$ for ${ }^{111} \mathrm{In}$-lowRLP, $14.9 \% \pm 4.1 \% \mathrm{ID} / \mathrm{g}$ for ${ }^{111} \mathrm{In}-\mathrm{medRLP}$, and somewhat lower for highRLP $(7.1 \% \pm 3.2 \% \mathrm{ID} / \mathrm{g})$ (Figure 4$)$.

In contrast to these findings, for ${ }^{111}$ In-medRLP with no added PEGylation blood levels at 1 hour postinjection were low with $5.0 \% \pm 0.9 \% \mathrm{ID} / \mathrm{g}$, whereas the spleen and liver uptake were the highest of all tested RLPs with $25.4 \% \pm 8.3 \%$ $\mathrm{ID} / \mathrm{g}$ for the spleen and $63.6 \% \pm 9.6 \% \mathrm{ID} / \mathrm{g}$ for the liver, respectively (Figure 4).

At 4 hours postinjection, blood levels had decreased and the uptake in the liver and spleen had increased to $50.6 \% \pm 11.1 \% \mathrm{ID} / \mathrm{g}$ for ${ }^{111} \mathrm{In}-\mathrm{lowRLP}, 56.1 \% \pm 2.2 \% \mathrm{ID} / \mathrm{g}$ for ${ }^{111} \mathrm{In}$-medRLP, and $30.5 \% \pm 8.0 \% \mathrm{ID} / \mathrm{g}$ for ${ }^{111} \mathrm{In}$-highRLP.

As shown in Figure 5, uptake in the receptor-positive tumor was $<1.0 \% \mathrm{ID} / \mathrm{g}$ for all RLPs, and no significant differences in uptake values between the M21 and the negative control M21-L tumor were found.

\section{Micro-SPECT/CT imaging studies}

In contrast to biodistribution studies, small animal imaging studies require administration of higher activities in order to obtain useful images. Therefore, approximately $12 \mathrm{MBq}$ of ${ }^{111}$ In-labeled RLPs (lowRLP, medRLP, and highRLP) were injected to each mouse.

In general, in vivo micro-SPECT/CT imaging of RLPs in beige severe combined immunodeficiency mice bearing U-87 MG tumor xenografts (left flank) showed similar biodistribution to the ex vivo biodistribution studies with M21/M21-L tumor xenografts, with accumulation of activity in the liver, spleen, and intestine (4 hours postinjection). The images demonstrated similar distribution patterns for ${ }^{111} \mathrm{In}$-lowRLP (Figure 6A) and ${ }^{111} \mathrm{In}$-medRLP (Figure 6B). ${ }^{111}$ In-highRLP (Figure 6C) showed a higher uptake in the spleen compared to the other two RLPs. The nonspecific uptake in the other organs (heart, stomach, pancreas, kidneys) was very low for all RLPs. The receptor-positive tumor was not clearly visualized at 4 hours postinjection and uptake did not increase over time.

\section{Discussion}

The use of LNPs to actively deliver agents to diseaseassociated cells (tumor sites) based upon molecular interaction with cell-surface receptors is an attractive diagnostic and therapeutic approach. Good results have already been achieved, especially in combination with targeting moieties such as RGD peptides and those derivatized with PEG to improve pharmacokinetics. ${ }^{35-37}$ 
Table I Biodistribution data of M2I/M2I-L tumor-bearing nude mice of different "'IIn-labeled RLPs

\begin{tabular}{|c|c|c|c|c|c|c|c|}
\hline & \multicolumn{4}{|c|}{$\%$ ID/g I hour postinjection } & \multicolumn{3}{|c|}{$\%$ ID/g 4 hours postinjection } \\
\hline & $\begin{array}{l}\text { II'In- } \\
\text { lowRLP }\end{array}$ & $\begin{array}{l}\text { "'In- } \\
\text { medRLP }\end{array}$ & $\begin{array}{l}\text { "''In- } \\
\text { highRLP }\end{array}$ & $\begin{array}{l}\text { I'In- } \\
\text { medRLP } \\
\text { (no PEG) }\end{array}$ & $\begin{array}{l}\text { I'In- } \\
\text { lowRLP }\end{array}$ & $\begin{array}{l}\text { "'In- } \\
\text { medRLP }\end{array}$ & $\begin{array}{l}\text { I'IIn- } \\
\text { highRLP }\end{array}$ \\
\hline Blood & $18.06 \pm 2.92$ & $17.04 \pm 5.14$ & $10.01 \pm 4.66$ & $5.03 \pm 0.93$ & $5.62 \pm 1.05$ & $4.53 \pm 1.09$ & $5.43 \pm 3.44$ \\
\hline Heart & $7.68 \pm 2.13$ & $8.30 \pm 2.43$ & $4.70 \pm 2.12$ & $2.32 \pm 0.54$ & $3.59 \pm 0.45$ & $2.58 \pm 0.95$ & $2.99 \pm 1.55$ \\
\hline Lung & $13.12 \pm 1.94$ & $14.88 \pm 4.14$ & $7.14 \pm 3.25$ & $3.57 \pm 0.85$ & $9.11 \pm 1.16$ & $5.54 \pm 1.31$ & $5.33 \pm 2.28$ \\
\hline Stomach & $1.26 \pm 0.59$ & $1.03 \pm 0.14$ & $0.90 \pm 0.45$ & $0.66 \pm 0.42$ & $1.08 \pm 0.40$ & $0.52 \pm 0.30$ & $1.13 \pm 0.74$ \\
\hline Pancreas & $\mathrm{I} .4 \mathrm{I} \pm 0.49$ & $1.90 \pm 1.04$ & $0.79 \pm 0.45$ & $1.33 \pm 1.00$ & $0.82 \pm 0.08$ & $0.59 \pm 0.16$ & $0.83 \pm 0.4 I$ \\
\hline Intestine & $2.98 \pm 0.43$ & $3.78 \pm 0.36$ & $1.68 \pm 0.89$ & $1.00 \pm 0.25$ & $8.90 \pm 3.32$ & $4.47 \pm 1.94$ & $4.58 \pm 2.00$ \\
\hline Spleen & $17.5 \mid \pm 5.12$ & $22.08 \pm 9.39$ & $18.19 \pm 9.25$ & $25.36 \pm 8.25$ & $12.37 \pm 3.77$ & $7.60 \pm 1.73$ & $18.05 \pm 4.70$ \\
\hline Liver & $32.95 \pm 5.67$ & $45.86 \pm 2.15$ & $21.52 \pm 6.54$ & $63.64 \pm 9.60$ & $50.62 \pm 11.10$ & $56.14 \pm 2.21$ & $30.54 \pm 8.04$ \\
\hline Kidneys & $5.09 \pm 1.31$ & $5.67 \pm 0.83$ & $7.40 \pm 5.69$ & $2.63 \pm 0.80$ & $5.45 \pm 1.63$ & $2.96 \pm 0.47$ & $8.05 \pm 4.19$ \\
\hline Muscle & $0.70 \pm 0.20$ & $1.23 \pm 0.39$ & $0.76 \pm 0.52$ & $0.22 \pm 0.15$ & $0.65 \pm 0.23$ & $0.37 \pm 0.07$ & $0.52 \pm 0.56$ \\
\hline M2I & $0.55 \pm 0.09$ & $0.69 \pm 0.03$ & $0.63 \pm 0.28$ & $0.33 \pm 0.07$ & $0.94 \pm 0.62$ & $0.38 \pm 0.13$ & $1.04 \pm 0.63$ \\
\hline M2I-L & $0.52 \pm 0.13$ & $0.59 \pm 0.08$ & $0.49 \pm 0.26$ & $0.31 \pm 0.05$ & $0.75 \pm 0.17$ & $0.25 \pm 0.04$ & $1.90 \pm 0.20$ \\
\hline
\end{tabular}

Note: Values are expressed as \% ID/g (mean \pm standard deviation) at I hour and 4 hours postinjection.

Abbreviations: \% ID/g, percentage injected dose per gram of tissue; "'In, indium-III; lowRLP, RLP with low RGD loading; highRLP, RLP with high RGD loading; medRLP, RLP with medium RGD loading; PEG, polyethylene glycol; RGD, arginyl-glycyl-aspartic acid; RLP, liposomal nanoparticles carrying an RGD building block.

The current study investigated LNPs carrying RGD and PEG building blocks and their ability to target $\alpha_{v} \beta_{3}$ integrin receptors overexpressed during tumor-induced angiogenesis. Several ${ }^{111}$ In-labeled RLPs derivatized with varying amounts of RGD and PEG building blocks were prepared to investigate their performance in vitro and in vivo using biodistribution experiments and micro-SPECT/CT imaging studies.

Until now, research on both in vivo and in vitro properties of targeted liposomes has been limited and concentrated on different targeting moieties. ${ }^{38,39}$ Previous studies have described the influence of PEG loading on targeting properties in vivo; ${ }^{7}$

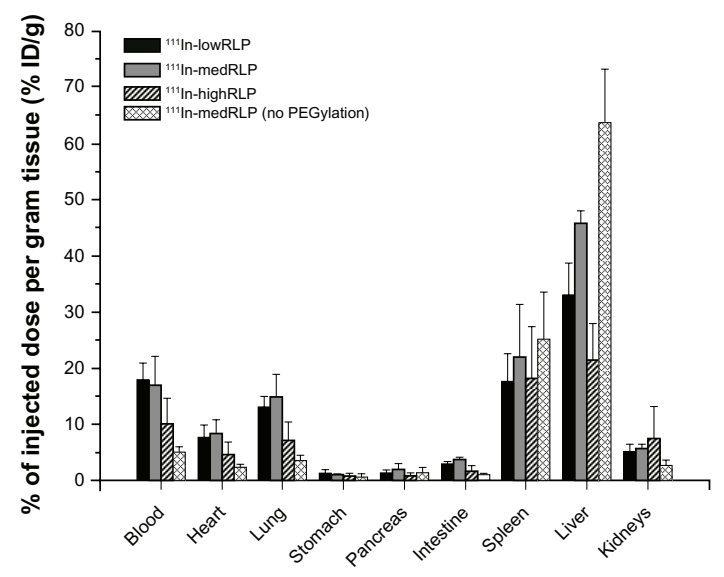

Figure 4 Comparison of biodistribution data of "'In-labeled lowRLP, medRLP, highRLP, and medRLP without added PEGylation using M2I/M2I-L tumor-bearing nude mice (I hour postinjection).

Note: Values are expressed as \% ID/g (mean \pm standard deviation).

Abbreviations: \% ID/g, percentage injected dose per gram of tissue; "'In, indium-I I I; lowRLP, RLP with low RGD loading; highRLP, RLP with high RGD loading; medRLP, RLP with medium RGD loading; RGD, arginyl-glycyl-aspartic acid; RLP, liposomal nanoparticles carrying an RGD building block. therefore, it was decided to investigate this behavior in vitro with RLPs carrying varying amounts of PEG. In vitro binding to isolated $\alpha_{v} \beta_{3}$ integrin receptors revealed the highest binding when no PEG building block was used (Figure 3 ) and binding decreased with higher PEG loading of the RLPs. Given the fact that Torchilin et al showed that higher PEG loading (10 mol\%) of immunoliposomes caused steric hindrance and resulted in lower accumulation in the target tissue in vivo, the same phenomena might well occur in vitro. ${ }^{40}$

On the other hand, PEGylation is advantageous when using LNPs in vivo as it prolongs their circulation time and reduces

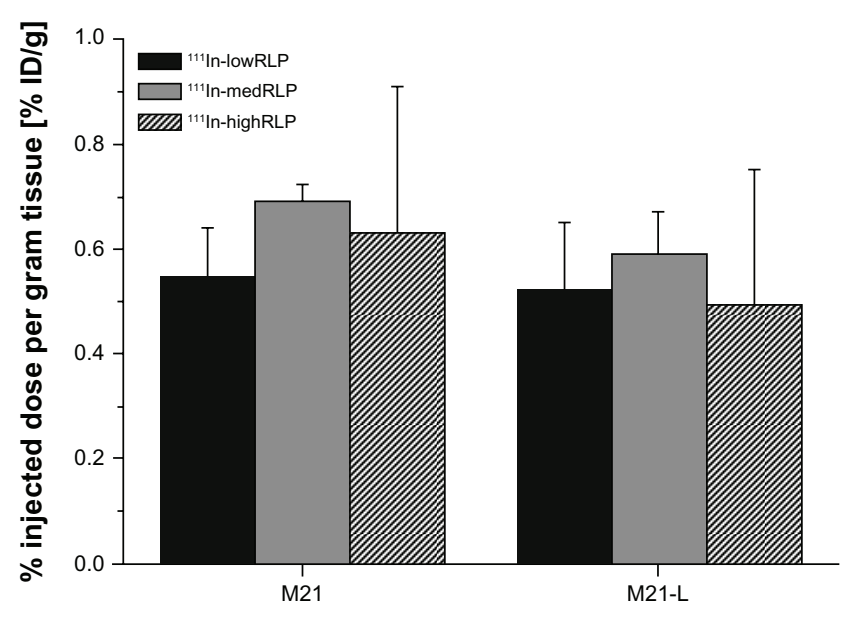

Figure 5 Ex vivo tumor uptake values of "'In-labeled lowRLP, medRLP, and highRLP in the M2I/M2I-L tumor xenograft model (I hour postinjection). Note: Values are expressed as \% ID/g (mean \pm standard deviation).

Abbreviations: \% ID/g, percentage injected dose per gram tissue; ' 'In, indium- I I I; lowRLP, RLP with low RGD loading; highRLP, RLP with high RGD loading; medRLP, RLP with medium RGD loading; RGD, arginyl-glycyl-aspartic acid; RLP, liposomal nanoparticles carrying an RGD building block. 


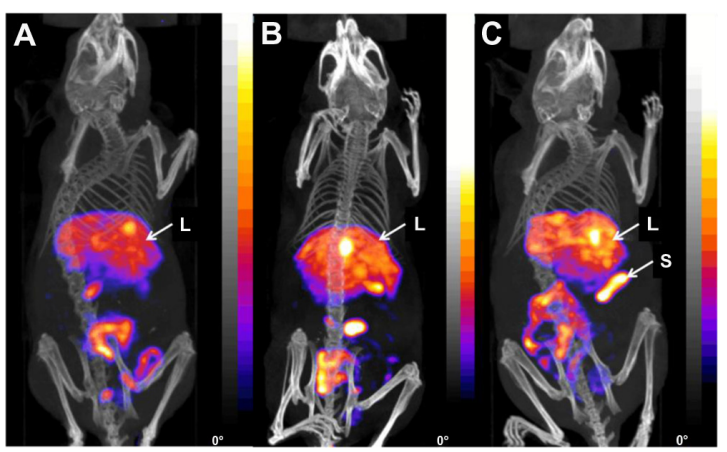

Figure 6 Fused micro single photon emission computed tomography/computed tomography images of three beige severe combined immunodeficiency mice with U-87 MG tumor xenografts on the left flank (4 hours postinjection) showing (A) "'In-labeled lowRLP, (B) "''In-labeled medRLP, and (C) "'In-labeled highRLP. Notes: All "'In-labeled RLPs showed main uptake in the liver, spleen, and intestine. For "'In-highRLP, uptake in the spleen was higher compared to the other two RLPs. Animals were placed in the anterior prone position (20 projections, 30 minutes).

Abbreviations: "'In, indium-III; L, liver; lowRLP, RLP with low RGD loading; highRLP, RLP with high RGD loading; medRLP, RLP with medium RGD loading; RGD, arginyl-glycyl-aspartic acid; RLP, liposomal nanoparticles carrying an RGD building block; S, spleen.

recognition by the mononuclear phagocyte system, giving the liposome sufficient time to arrive at its target. ${ }^{41}$ This hypothesis was confirmed in the M21/M21-L tumor xenograft model. When injected into nude mice, blood levels of ${ }^{111}$ In-medRLP with no PEG building block were much lower than those of ${ }^{11}$ In-medRLP with PEGylation. High blood levels are an indication of in vivo stability and are desired since these RLPs are designed to be used as carriers for different drugs that need prolonged circulation times. But in this case, it has to be considered that the RLPs carry the RGD-targeting sequence attached to the end of the PEG chain. If so, it is clear that the amount of PEG connected to the RGD building block was not sufficient for the pronounced stealth effect of the liposome.

Concerning the effect of peptide loading of liposomes, initial studies with a tyrosine-3-octreotide peptide attached to LNPs have already been performed by the authors' group with promising results. ${ }^{22}$ Based on these findings, the different RLPs were evaluated with respect to their RGD loading. In the competition assay, the half-maximal inhibitory values of the RLPs showed a clear relationship between RGD loading and replacement of ${ }^{125}$ I-cyclo-(-RGDyV-) at the receptor. Almost no competition was observed for lowRLP, whereas the higher loading of medRLP and highRLP decreased the concentration of RLP required to replace ${ }^{125}$ I-cyclo-(-RGDyV-) by a factor of about six. As expected, no binding to the isolated receptors and therefore no replacement was observed in the nonderivatized standardLP.

When the total binding of ${ }^{111}$ In-labeled RLPs was determined, higher RGD loading led to significantly higher binding to the $\alpha_{\mathrm{v}} \beta_{3}$ integrin receptors (Figure 2). This is in agreement with the results of Du et al who found an RGD dose-dependent uptake in hepatic stellate cells and an increased inhibition of hepatic stellate cell proliferation with cyclic RGD sterically stabilized liposomes. ${ }^{42}$ The specific uptake in hepatic stellate cells increased with higher RGD loading of the sterically stabilized liposomes, which could be beneficial in treating liver fibrosis. These findings are in accordance with Shokeen et al who used multivalent, functional polymer nanoparticles (CNP) derivatized with varying amounts of a linear RGD peptide $(5 \%, 10 \%, 20 \%$, $50 \%$ RGD loading).$^{43}$ The results of binding assays on isolated integrins demonstrated an approximate 75 -fold increase in binding between 5\% RGD-CNP and 50\% RGD-CNP. In contrast to that, Jiang et al observed optimal cellular transfection and tumor inhibition in a drug-resistant MCF7/A tumor with 1\% RGD-modified liposomes containing small interfering ribonucleic acid, suggesting that the $1 \%$ molar ratio of RGD modification might be the best ratio for targeting integrin receptors. ${ }^{44}$

Nevertheless, biodistribution studies of ${ }^{111}$ In-labeled RLPs did not confirm the in vitro data. In general, the highest uptake of RLPs was in the liver and spleen, which was expected since LNPs accumulate in these organs due to extraction via the mononuclear phagocyte system. The tumor targeting characteristics were suboptimal, with an uptake in the $\alpha_{v} \beta_{3}$ integrin receptor-positive M21 tumor of less than 1\% ID/g (Figure 5). Although tumor uptake values of the M21 tumors were slightly higher than those of the negative control M21-L tumors (with lowest values for lowRLP), the differences were not significant. This outcome was not as anticipated since the in vitro behavior of RLPs seemed to be promising and the authors' group has already demonstrated a specific tumor binding of $2.5 \% \mathrm{ID} / \mathrm{g}$ with tyrosine-3-octreotide liposomes, ${ }^{22}$ which is comparable to the data of Accardo et al. ${ }^{45}$ An even better tumor uptake of $5.2 \% \mathrm{ID} / \mathrm{g}$ in mice bearing a somatostatin receptor-2positive human neuroendocrine tumor was found for copper64-labeled octreotate-encapsulated liposomes. ${ }^{25}$ Reasons for the reduced tumor targeting of RLPs might be related to the PEG(2000) chain length as reported by Herringson and Altin. ${ }^{11}$ They suggested that the long PEG(2000) chains disrupt the interaction of liposome-anchored moieties with their receptors and that shorter PEG chains may be advantageous as they would interfere less with binding sites on the tumor. Another reason could be that the $\alpha_{v} \beta_{3}$ integrin receptors of the solid tumors used in the current xenograft model are not as easily accessible as $\alpha_{v} \beta_{3}$ integrins 
of other tumors or, for example, special antigens located on the vascular endothelial cell surface. ${ }^{46}$ Lehtinen et al additionally stated that PEGylated liposomes are efficiently taken up at the tumor site by the enhanced permeability and retention effect but it might be possible that active targeting is not visible in a conventional biodistribution study. ${ }^{47}$ In accordance with these results, specific targeting of RLPs to the tumors could not be visualized using micro-SPECT/ CT imaging, but a similar accumulation pattern was seen compared to the ex vivo biodistribution studies.

Overall, the experimental results did not show any evidence of specific tumor targeting of RLPs in vivo. As mentioned above, there could be various reasons for this and these results emphasize how challenging liposomal targeting approaches are. For optimal uptake, the ideal concentration of targeting sequences, PEG loading, and also particle formation must be determined. In particular, if the concentration of the targeting moiety is too high, this could lead to an abnormal mononuclear phagocyte system response followed by fast blood clearance of the LNP. ${ }^{43}$ Montet et al postulated that there is an upper limit for RGD loading and that this limit is reached if the number of RGD peptides on the LNP exceeds that of the receptor binding sites on the cell membrane. ${ }^{48}$

An additional challenge is to choose the optimal injected lipid dose when in vivo targeting is attempted. On the one hand the amount of LNP should be low enough to avoid receptor saturation, on the other hand it has been shown by Laverman et al that circulation times may be reduced and liver uptake increased at very low lipid doses. ${ }^{49}$ In the current study, a lipid dose of about $0.5 \mu \mathrm{mol} / \mathrm{kg}$ bodyweight was injected, a dose where this effect has not been reported. Although the total amount of injected RGD varied, depending on the LNP composition, this cannot explain the overall low tumor uptake in vivo.

It may also be argued that ${ }^{111} \mathrm{In}$ is not bound with sufficient stability to the LNP or the LNP itself might not be stable after intravenous injection, resulting in loss of targeting. However, extensive studies have been carried out on the radiolabeling properties and stability of LNPs (also labeled with different radiometals via this approach), which have shown high in vitro stability of the LNPs in competition with aqueous solution, human serum, and DTPA, as well as high blood pool retention in vivo. ${ }^{22}$ Therefore, the stability seems to be sufficient to achieve targeting in vivo.

Another parameter influencing targeting properties and in vivo stability is the size and charge of the LNP. The NPs used in this study only showed a slight variation in size between
90-110 nm and no significant differences in zeta potential. But zeta potentials slightly shifted towards a higher positive charge with increased RGD loading, as would be expected. Nevertheless, these differences both regarding size and charge were not pronounced and it seems unlikely that they could account for the observed limited in vivo targeting. It has been suggested that higher negative zeta potential values may have a positive effect on stability, ${ }^{50}$ and both slightly negative and positive surface charges improve blood circulation kinetics. ${ }^{51}$ Extensive evaluation of the influence of charge for targeting applications should be a topic of future investigations.

Overall studies of the use of liposomes carrying RGD peptides as the targeting moiety are encouraging as these liposomes loaded with paclitaxel (a widely used antimicrotubule agent for the treatment of a variety of cancers) have been shown to have antitumor activity. ${ }^{52}$ They have also been used for selective cell targeting to treat cardiovascular disease such as atherosclerosis, restenosis, or inflammation processes. ${ }^{53}$

Future experiments will have to investigate the ideal balance of RGD and PEG loading. Since both the in vitro and in vivo experiments showed that PEG has a pronounced influence on the pharmacokinetics of the liposome and that its presence increases the circulation time in blood, varying PEG lengths or even finding alternatives to PEG, as proposed by Knop et al, ${ }^{54}$ should be considered. Taking into account that PEG influences binding to tumor sites, further studies could include relocating the RGD derivatization to the PEG building block and varying the amount of RGD loading of the liposome.

\section{Conclusion}

This study investigated the influence of PEGylation and RGD loading of different LNPs in relation to their targeting properties, in particular to visualize tumor-induced angiogenesis. Even though the influence of PEG loading was shown and good binding to isolated $\alpha_{v} \beta_{3}$ integrin receptors in vitro was found (which could be improved with higher RGD loading), these results could not be confirmed in vivo with biodistribution and micro-SPECT/CT imaging studies. Consequently, an optimized balance between PEGylation and RGD loading needs to be found to achieve ideal specific tumor targeting in vivo. Nonetheless, the results of this work still show that LNPs are an attractive tool for targeting strategies. Since new developments in nanotechnology and also molecular biology are increasing, an ideal multimodal nanoparticle featured with different types of ligands used 
for diagnosis, imaging, and therapy is much needed in the future.

\section{Acknowledgments}

Dr David A Cheresh and the Scripps Research Institute (La Jolla, CA) are acknowledged for providing the human melanoma M21 and M21-L cells. Jerome Burnet, Ciara Finucane, and Julie Foster assisted in the microSPECT/ CT imaging studies carried out at the Center for Molecular Oncology, Barts Cancer Institute, Queen Mary University of London. The authors would like to express their thanks to Glen Perera, Institute of Pharmacy, University of Innsbruck, for assistance with the zeta potential measurements. The Austrian Nano-Initiative cofinanced this work as part of the Nano-Health project (number 0200), the subprojects NANO-NUC were financed by the Austrian FWF (Fonds zur Forderung der Wissenschaftlichen Forschung; number N208-NAN), and NANO-LIPO was financed by the Austrian Research Promotion Agency FFG (project Nano-Health number 819721).

\section{Disclosure}

The authors report no conflicts of interest in this work.

\section{References}

1. Bangham AD, Standish MM, Watkins JC. Diffusion of univalent ions across the lamellae of swollen phospholipids. J Mol Biol. 1965;13(1): 238-252.

2. Maurer N, Fenske DB, Cullis PR. Developments in liposomal drug delivery systems. Expert Opin Biol Ther. 2001;1(6):923-947.

3. Boerman OC, Laverman P, Oyen WJ, Corstens FH, Storm G. Radiolabeled liposomes for scintigraphic imaging. Prog Lipid Res. 2000; 39(5):461-475.

4. Allen TM, Cullis PR. Drug delivery systems: entering the mainstream. Science. 2004;303(5665):1818-1822.

5. Duncan R, Gaspar R. Nanomedicine(s) under the microscope. Mol Pharm. 2011;8(6):2101-2141.

6. Gabizon AA. Stealth liposomes and tumor targeting: one step further in the quest for the magic bullet. Clin Cancer Res. 2001;7(2): 223-225.

7. Li SD, Huang L. Stealth nanoparticles: high density but sheddable PEG is a key for tumor targeting. J Control Release. 2010;145(3):178-181.

8. Tirosh O, Barenholz Y, Katzhendler J, Priev A. Hydration of polyethylene glycol-grafted liposomes. Biophys J. 1998;74(3):1371-1379.

9. Zhao W, Zhuang S, Qi XR. Comparative study of the in vitro and in vivo characteristics of cationic and neutral liposomes. Int J Nanomedicine. 2011;6:3087-3098.

10. Gref R, Luck M, Quellec P, et al. 'Stealth' corona-core nanoparticles surface modified by polyethylene glycol (PEG): influences of the corona (PEG chain length and surface density) and of the core composition on phagocytic uptake and plasma protein adsorption. Colloids Surf B Biointerfaces. 2000;18(3-4):301-313.

11. Herringson TP, Altin JG. Effective tumor targeting and enhanced antitumor effect of liposomes engrafted with peptides specific for tumor lymphatics and vasculature. Int J Pharm. 2011;411(1-2):206-214.

12. Torchilin VP. Tumor delivery of macromolecular drugs based on the EPR effect. Adv Drug Deliv Rev. 2011;63(3):131-135.
13. Lattin JR, Belnap DM, Pitt WG. Formation of eLiposomes as a drug delivery vehicle. Colloids Surf B Biointerfaces. 2012;89:93-100.

14. Ashley CE, Carnes EC, Phillips GK, et al. The targeted delivery of multicomponent cargos to cancer cells by nanoporous particle-supported lipid bilayers. Nat Mater. 2011;10(5):389-397.

15. Li ZB, Chen K, Chen X. (68)Ga-labeled multimeric RGD peptides for microPET imaging of integrin $\alpha(\mathrm{v}) \beta(3)$ expression. Eur $J$ Nucl Med Mol Imaging. 2008;35(6):1100-1108.

16. Barrett T, Brechbiel M, Bernardo M, Choyke PL. MRI of tumor angiogenesis. J Magn Reson Imaging. 2007;26(2):235-249.

17. Ferrara N, Kerbel RS. Angiogenesis as a therapeutic target. Nature. 2005;438(7070):967-974.

18. Weidner N, Semple JP, Welch WR, Folkman J. Tumor angiogenesis and metastasis - correlation in invasive breast carcinoma. NEngl J Med. 1991;324(1):1-8.

19. Griffioen AW, Molema G. Angiogenesis: potentials for pharmacologic intervention in the treatment of cancer, cardiovascular diseases, and chronic inflammation. Pharmacol Rev. 2000;52(2):237-268.

20. Haubner R, Wester HJ, Weber WA, et al. Noninvasive imaging of alpha(v)beta3 integrin expression using 18F-labeled RGD-containing glycopeptide and positron emission tomography. Cancer Res. 2001; 61(5):1781-1785.

21. Desgrosellier JS, Cheresh DA. Integrins in cancer: biological implications and therapeutic opportunities. Nat Rev Cancer. 2010;10(1): 9-22.

22. Helbok A, Decristoforo C, Dobrozemsky G, et al. Radiolabeling of lipid-based nanoparticles for diagnostics and therapeutic applications: a comparison using different radiometals. J Liposome Res. 2010;20(3):219-227.

23. Marik J, Tartis MS, Zhang H, et al. Long-circulating liposomes radiolabeled with [18F]fluorodipalmitin ([18F]FDP). Nucl Med Biol. 2007;34(2):165-171.

24. Tsai CC, Chang $\mathrm{CH}$, Chen LC, et al. Biodistribution and pharmacokinetics of 188Re-liposomes and their comparative therapeutic efficacy with 5-fluorouracil in $\mathrm{C} 26$ colonic peritoneal carcinomatosis mice. Int J Nanomedicine. 2011;6:2607-2619.

25. Petersen AL, Binderup T, Jolck RI, et al. Positron emission tomography evaluation of somatostatin receptor targeted 64Cu-TATE-liposomes in a human neuroendocrine carcinoma mouse model. J Control Release. 2012;160(2):254-263.

26. Jonasdottir TJ, Fisher DR, Borrebaek J, Bruland OS, Larsen RH. First in vivo evaluation of liposome-encapsulated 223Ra as a potential alpha-particle-emitting cancer therapeutic agent. Anticancer Res. 2006;26(4B):2841-2848.

27. Henriksen G, Schoultz BW, Michaelsen TE, Bruland OS, Larsen RH. Sterically stabilized liposomes as a carrier for alpha-emitting radium and actinium radionuclides. Nucl Med Biol. 2004;31(4):441-449.

28. Bard DR, Knight CG, Page-Thomas DP. Effect of the intra-articular injection of lutetium-177 in chelator liposomes on the progress of an experimental arthritis in rabbits. Clin Exp Rheumatol. 1985;3(3): $237-242$.

29. McQuarrie S, Mercer J, Syme A, Suresh M, Miller G. Preliminary results of nanopharmaceuticals used in the radioimmunotherapy of ovarian cancer. J Pharm Pharm Sci. 2005;7(4):29-34.

30. Mougin-Degraef M, Bourdeau C, Jestin E, et al. Doubly radiolabeled liposomes for pretargeted radioimmunotherapy. Int J Pharm. 2007; 344(1-2):110-117.

31. Rojas S, Gispert JD, Martin R, et al. Biodistribution of amino-functionalized diamond nanoparticles. In vivo studies based on 18F radionuclide emission. ACS Nano. 2011;5(7):5552-5559.

32. Nachman RL, Leung LL. Complex formation of platelet membrane glycoproteins IIb and IIIa with fibrinogen. J Clin Invest. 1982;69(2): 263-269.

33. Fraker PJ, Speck JC Jr. Protein and cell membrane iodinations with a sparingly soluble chloroamide, 1,3,4,6-tetrachloro-3 $\alpha, 6 \alpha$ diphenylglycoluril. Biochem Biophys Res Commun. 1978;80(4): 849-857. 
34. Haubner R, Weber WA, Beer AJ, et al. Noninvasive visualization of the activated alphavbeta3 integrin in cancer patients by positron emission tomography and [18F]Galacto-RGD. PLoS Med. 2005;2(3):e70.

35. Su W, Wang H, Wang S, et al. PEG/RGD-modified magnetic polymeric liposomes for controlled drug release and tumor cell targeting. Int $J$ Pharm. 2012;426(1-2):170-181.

36. Holig P, Bach M, Volkel T, et al. Novel RGD lipopeptides for the targeting of liposomes to integrin-expressing endothelial and melanoma cells. Protein Eng Des Sel. 2004;17(5):433-441.

37. Qin J, Chen D, Hu H, Qiao M, Zhao X, Chen B. Body distribution of RGD-mediated liposome in brain-targeting drug delivery. Yakugaku Zasshi. 2007;127(9):1497-1501.

38. Helbok A, Rangger C, Von Guggenberg E, et al. Targeting properties of peptide-modified radiolabeled liposomal nanoparticles. Nanomedicine. 2012;8(1):112-118.

39. Falciani C, Accardo A, Brunetti J, et al. Target-selective drug delivery through liposomes labeled with oligobranched neurotensin peptides. Chem Med Chem. 2011;6(4):678-685.

40. Torchilin VP, Klibanov AL, Huang L, O’Donnell S, Nossiff ND, Khaw BA. Targeted accumulation of polyethylene glycol-coated immunoliposomes in infarcted rabbit myocardium. FASEB J. 1992; 6(9):2716-2719.

41. Li S, Goins B, Zhang L, Bao A. Novel multifunctional theranostic liposome drug delivery system: construction, characterization, and multimodality MR, near-infrared fluorescent, and nuclear imaging. Bioconjug Chem. May 22, 2012 . [Epub ahead of print.]

42. Du SL, Pan H, Lu WY, Wang J, Wu J, Wang JY. Cyclic Arg-Gly-Asp peptide-labeled liposomes for targeting drug therapy of hepatic fibrosis in rats. J Pharmacol Exp Ther. 2007;322(2):560-568.

43. Shokeen M, Pressly ED, Hagooly A, et al. Evaluation of multivalent, functional polymeric nanoparticles for imaging applications. ACS Nano. 2011;5(2):738-747.

44. Jiang J, Yang SJ, Wang JC, et al. Sequential treatment of drugresistant tumors with RGD-modified liposomes containing siRNA or doxorubicin. Eur J Pharm Biopharm. 2010;76(2):170-178.
45. Accardo A, Mansi R, Morisco A, et al. Peptide modified nanocarriers for selective targeting of bombesin receptors. Mol Biosyst. 2010;6(5): 878-887.

46. Mori A, Klibanov AL, Torchilin VP, Huang L. Influence of the steric barrier activity of amphipathic poly(ethyleneglycol) and ganglioside GM1 on the circulation time of liposomes and on the target binding of immunoliposomes in vivo. FEBS Lett. 1991;284(2): 263-266.

47. Lehtinen J, Magarkar A, Stepniewski M, et al. Analysis of cause of failure of new targeting peptide in PEGylated liposome: molecular modeling as rational design tool for nanomedicine. Eur J Pharm Sci. 2012;46(3):121-130.

48. Montet X, Funovics M, Montet-Abou K, Weissleder R, Josephson L. Multivalent effects of RGD peptides obtained by nanoparticle display. J Med Chem. 2006;49(20):6087-6093.

49. Laverman P, Brouwers AH, Dams ET, et al. Preclinical and clinical evidence for disappearance of long-circulating characteristics of polyethylene glycol liposomes at low lipid dose. J Pharmacol Exp Ther. 2000;293(3):996-1001.

50. Maherani B, Arab-Tehrany E, Kheirolomoom A, Reshetov V, Stebe MJ, Linder M. Optimization and characterization of liposome formulation by mixture design. Analyst. 2012;137(3):773-786.

51. Brinkhuis RP, Stojanov K, Laverman P, et al. Size dependent biodistribution and SPECT imaging of (111)In-labeled polymersomes. Bioconjug Chem. April 12, 2012. [Epub ahead of print.]

52. Zhao H, Wang JC, Sun QS, Luo CL, Zhang Q. RGD-based strategies for improving antitumor activity of paclitaxel-loaded liposomes in nude mice xenografted with human ovarian cancer. $J$ Drug Target. 2009;17(1):10-18.

53. Lestini BJ, Sagnella SM, Xu Z, et al. Surface modification of liposomes for selective cell targeting in cardiovascular drug delivery. J Control Release. 2002;78(1-3):235-247.

54. Knop K, Hoogenboom R, Fischer D, Schubert US. Poly(ethylene glycol) in drug delivery: pros and cons as well as potential alternatives. Angew Chem Int Ed Engl. 2010;49(36):6288-6308.
International Journal of Nanomedicine

\section{Publish your work in this journal}

The International Journal of Nanomedicine is an international, peerreviewed journal focusing on the application of nanotechnology in diagnostics, therapeutics, and drug delivery systems throughout the biomedical field. This journal is indexed on PubMed Central, MedLine, CAS, SciSearch ${ }^{\circledR}$, Current Contents ${ }^{\circledR} /$ Clinical Medicine,

\section{Dovepress}

Journal Citation Reports/Science Edition, EMBase, Scopus and the Elsevier Bibliographic databases. The manuscript management system is completely online and includes a very quick and fair peer-review system, which is all easy to use. Visit http://www.dovepress.com/ testimonials.php to read real quotes from published authors. 